\title{
Significance of EZH2 and BMI-1 Protein Expression in Carcinogenesis of Colon Serrated Adenoma
}

\author{
Kai Zhao* \\ Department of Gastroenterology, Jintan Hospital, Jiangsu University, Changzhou 213200, Jiangsu Province, China \\ *Corresponding author: Kai Zhao, zhaokau@163.com
}

\begin{abstract}
Objective: To observe the significance of EZH2 and BMI-1 protein expression in the carcinogenesis process of colon serrated adenoma (SSA/P). Methods: Hematoxylin-eosin (HE) staining was used to observe the morphological characteristics of normal tissues, hyperplastic polyp (HP), SSA/P and colon cancer. Immunohistochemical staining was used to detect the expression of EZH2 and BMI-1 protein. The relative expression of EZH2 and BMI-1 was detected by qRT-PCR. Results: Compared with normal tissues, HP and colon cancer tissues, SSA/P showed serrated glandular hyperplasia, glandular dilatation, and deep nuclear staining, which had certain atypia. The positive expression rates of EZH2 and BMI-1 protein were $53.3 \%$ and $56 \%$, which were close to those of colon cancer $(66.7 \%$ and $76.6 \%)$ and higher than those of normal group and HP $(16 \%$ and $8 \%, \mathrm{P}<0.05)$. The relative expression of EZH2 and BMI-1 in SSA/P tissue was significantly higher than that in normal group and HP, but lower than that in carcinogenesis group $(\mathrm{P}<0.05)$. Conclusion: EZH2 and BMI-1 play an important role in the carcinogenesis of colon serrated adenoma, and can be used as the primary screening index before carcinogenesis.
\end{abstract}

Keywords: Serrated adenoma of colon; EZH2; BMI-1

Publication date: July 2021; Online publication: July 31, 2021

\section{Introduction}

Enhancer of zeste homolog 2 (EZH2) and B cell-specific Moloney murine leukemia virus integration site 1 (BMI-1) are important members of the Polycomb group ( $\mathrm{pcG}$ ) family and the main oncogenes. They promote the occurrence and development of tumors by catalyzing histone methylation, activating multiple signaling pathways, and promoting epithelial-mesenchymal cell transformation (EMT) ${ }^{[1]}$. EZH2 and BMI1 have been confirmed to be highly expressed in colorectal cancer tissues, and are closely related to tumor differentiation and metastasis ${ }^{[2]}$. However, the expression in the process of colorectal serrated adenoma carcinogenesis has not been studied yet. Therefore, this study aims to explore the expression of EZH2 and BMI-1 in the process of colorectal serrated adenoma carcinogenesis, and to explore its clinical significance.

\section{Methods and Materials}

\subsection{General materials}

From January 2019 to December 2020, patients who underwent endoscopic detection of gastrointestinal lesions in the Department of Gastroenterology, Jintan Hospital, Jiangsu University were selected and their tissue samples were taken. Among them, were 15 cases of normal mucosal tissue (Normal), 25 cases of hyperplastic polyp (HP), 30 cases of sessile serrated adenoma (SSA/P), and 30 cases of colorectal cancer tissue (Cancer). Part of the tissue taken was sent to pathology, and the remaining part was directly put into Eppendorf (EP) tube and frozen at $-80^{\circ} \mathrm{C}$ for later use. 


\subsection{Histopathological examination}

After embedding the tissue in paraffin, it was sliced to a thickness of $4 \mu \mathrm{m}$, and deparaffinized it with water as usual. The specific steps of immunohistochemistry were in accordance with the standard operating procedures. The primary antibody of EZH2 and BMI-1 was purchased from Abcam, and the secondary antibody was purchased from Nantong Biyuntian Biotechnology Co. Ltd. The result was judged as positive when the nucleus appeared claybank.

\subsection{Quantitative real-time PCR detection (qRT-PCR detection)}

The frozen tissues were extracted with TRIzol reagent for total mRNA, and cDNA was synthesized according to the standard operating procedures of first strand cDNA synthesis kit. The mRNA level was further analyzed in accordance with the standard operating instructions of the real-time quantitative PCR kit. EZH2 and BMI-1 primers were purchased from GenePharma, and the kits were purchased from Invitrogen, USA.

\section{Result}

3.1. HE staining for morphological characteristics of normal tissues, HP, SSA/P and colorectal cancerous tissues

In the observation of HE staining, compared with normal tissues, glandular hyperplasia could be seen in the lamina propria in HP, which is tubular, part of the lumen edge is serrated, and glandular epithelial cells are not atypia. In the entire crypt structure of SSA/P, there were serrated gland hyperplasia, gland expansion, and deep staining of the nucleus, with a certain atypia. Cancer is composed of densely arranged glands, some of the glands which have a serrated cavity surface, glandular epithelium is heterosexual, the glands merge into a sieve mesh, the glandular epithelium is severely atypia, and nuclear division and invasive growth could be seen (Figure 1.).
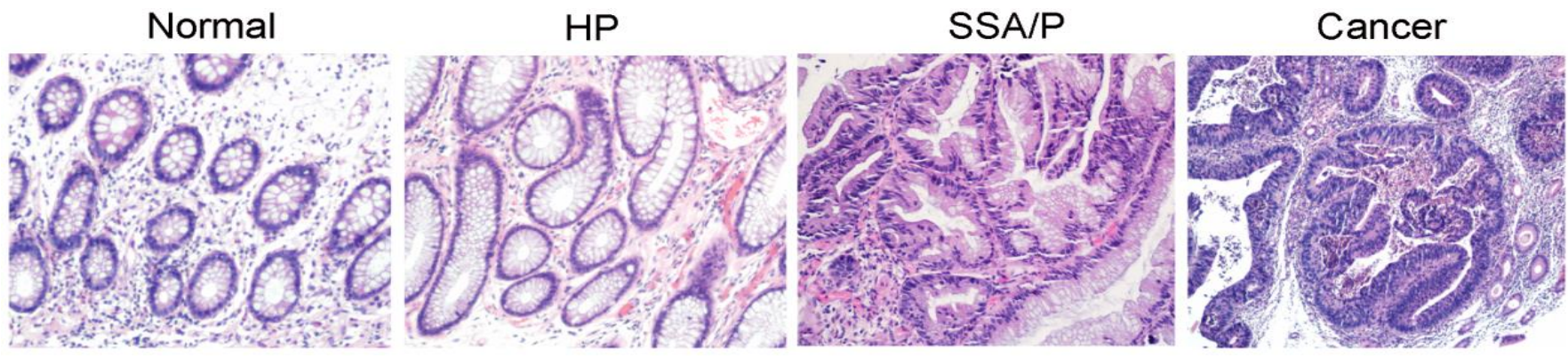

Figure 1. The morphological characteristics of HE staining of normal tissues, HP, SSA/P and colorectal cancerous tissues $(200 x)$

\subsection{Immunohistochemical detection of the expression of EZH2 and BMI-1 in HP, SSA/P and colorectal cancerous tissues}

The results of immunohistochemistry showed that EZH2 and BMI-1 were mainly expressed in the nucleus, and part of the cytoplasm was stained. In this study, EZH2 was not expressed in normal tissues, but four cases $(12 \%)$ were occasionally positively expressed in HP, 12 cases $(53.3 \%)$ were positively expressed in $\mathrm{SSA} / \mathrm{P}$, and 20 cases $(66.7 \%$ ) were positively expressed in colorectal cancer tissues. The difference between the three groups was statistically significant $(\mathrm{P}<0.05)$ (Table 1., Figure 2.). The positive expression scores of BMI-1 in HP, SSA/P, and colorectal cancerous tissues were $8 \%, 56 \%$, and $76.6 \%$. The difference between the three groups was statistically significant $(\mathrm{P}<0.05)$ (Table 1., Figure 2.). 
Table 1. Expression of EZH2 and BMI-1 in three tissues

\begin{tabular}{lccc}
\hline \multicolumn{1}{c}{ Groups } & n (number of cases) & EZH2 positive & BMI-1 positive \\
\hline Normal mucosal tissue (Normal) & 15 & $0(0.0)$ & $0(0.0)$ \\
Hyperplastic polyps (HP) & 25 & $3(16 \%)$ & $1(8.0 \%)$ \\
Sessile serrated adenoma (SSA/P) & 30 & $16(53.3 \%)$ & $14(56 \%)$ \\
Colorectal cancer tissue (Cancer) & 30 & $22(66.7 \%)$ & $23(76.7 \%)$ \\
\hline
\end{tabular}

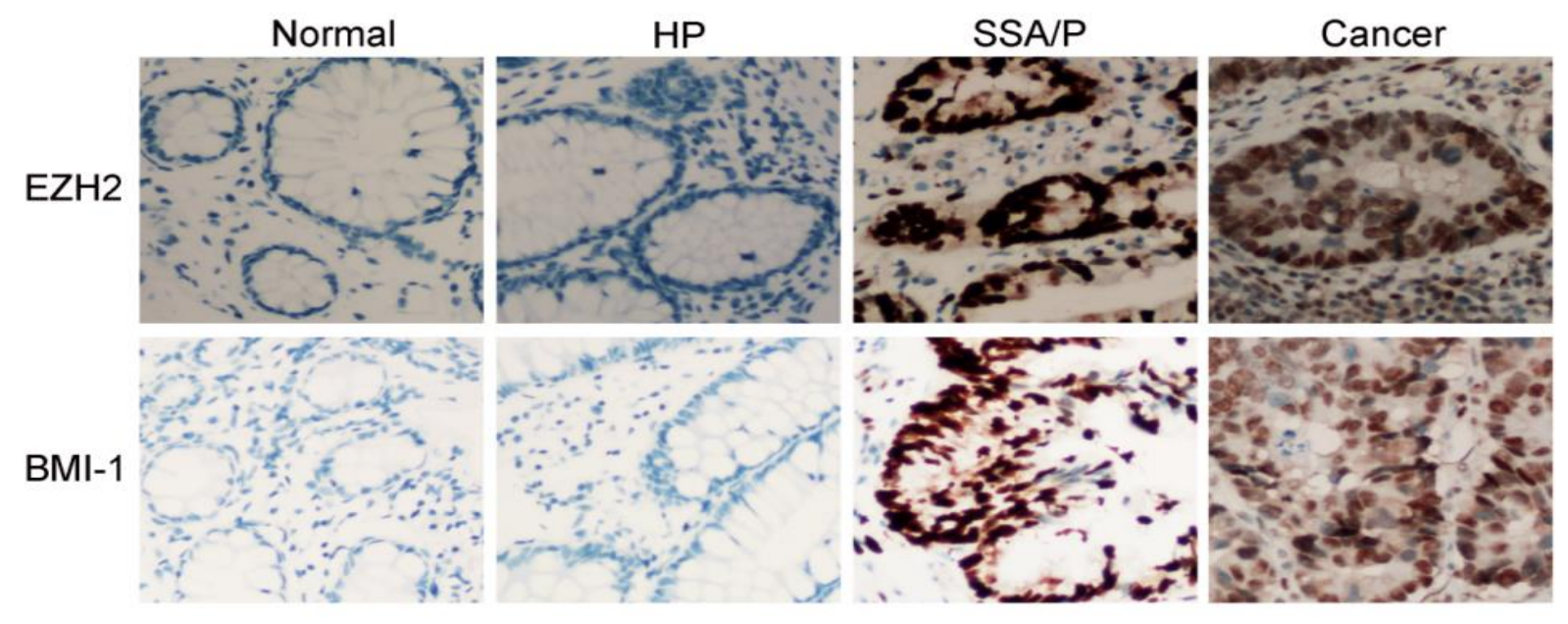

Figure 2. Expression of EZH2 and BMI-1 in tissues (200x)

\subsection{The relative expression of EZH2 and BMI-1 in HP, SSA/P and colorectal cancerous tissues}

The qRT-PCR results showed that, compared with the control group, the expressions of EZH2 and BMI-1 in the HP, SSA/P, and cancerous groups were significantly up-regulated, suggesting that EZH1 and BMI1 are closely related to the serrated carcinogenesis of the colorectal and increase with the increase of the degree of tissue malignancy (Figure 3.).

A

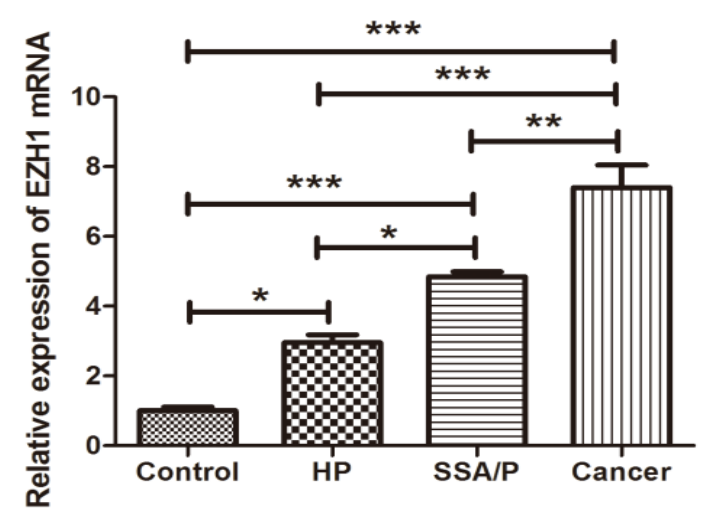

B

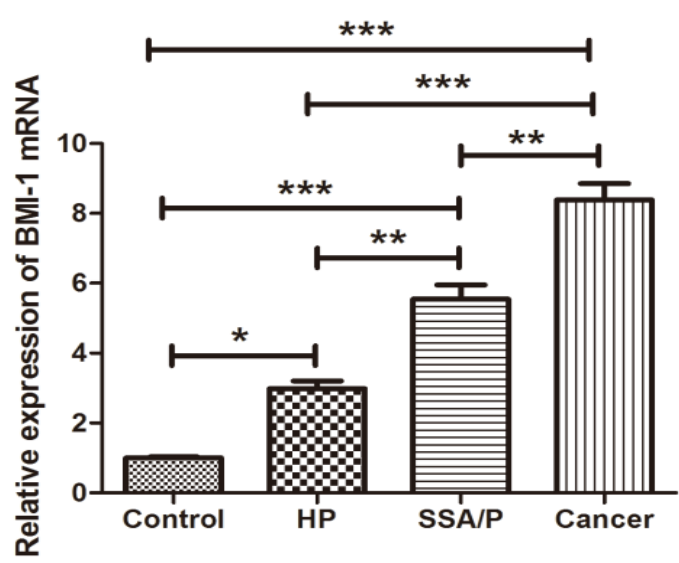

Figure 3. The expression of EZH1 and BMI-1 was detected by qRT-PCR

\section{Discussion}

Colorectal cancer is one of the common malignant tumors of the digestive tract ${ }^{[3]}$. In recent years, with the changes in the diet and lifestyle in China, the incidence of colorectal cancer has been increasing year by year, but the etiology is not yet fully understood. Serrated adenoma has been considered as a pre-stage pathway of colorectal cancer, which involves a series of genetic and epigenetic changes. With the 
continuous development of molecular biology, people gradually have a clear understanding of the genes of molecular events that occur in the process of cancer. Identifying the genes involved in the carcinogenesis of serrated adenomas can provide references for early diagnosis, treatment and prevention.

EZH2 and BMI-1 belong to the pcG family and play a central role in their family ${ }^{[1]}$. EZH2 is the human homologue of the enhancer of the Drosophila zeste gene. It can be used as a transcriptional blocking factor to reduce the binding to DNA, thereby inhibiting the expression of target genes, and promoting cell cycle evolution and cell malignancy ${ }^{[4]}$. BMI-1 is considered to be a proto-oncogene, which can cooperate with the oncogene c-myc to cause cell transformation and tumor formation ${ }^{[5]}$. In addition, EZH2 and BMI$1 \mathrm{can}$ form a polyprotein complex to modify the chromosomes to maintain the silencing of homeobox genes. This effect is closely related to cell differentiation, proliferation and control of transcription. Additionally, its excessive activation or inhibition will lead to the occurrence of tumors ${ }^{[6,7]}$. What's more, EZH2 and BMI-1 are positively correlated with the degree of malignancy and invasion of a variety of tumors, and have been used as sensitive indicators for early diagnosis and prognostic follow-up of certain tumors ${ }^{[1,8,9]}$. In this study, it was found that the results of immunohistochemistry and qRT-PCR showed that EZH1 and BMI-1 are closely related to the process of colorectal serrated carcinogenesis, and they increase with the increase of the degree of tissue malignancy. These suggest that EZH1 and BMI-1 are involved in the carcinogenesis of colorectal serrated adenoma. EZH1 and BMI-1 can be used as an early screening index for colorectal serrated adenoma cancer, which is of great significance for the prevention and treatment of colorectal.

\section{Disclosure statement}

The author declares no conflict of interest.

\section{References}

[1] Steele JC, Torr EE, Noakes KL, et al., 2006, The Polycomb Group Proteins, BMI-1 and EZH2, are Tumour-Associated Antigens. British Journal of Cancer.

[2] Bao H, Li J, Xing G, 2014, Expression and Significance of EZH2 and Bmi-1 Genes in Colorectal Cancer Tissues. Heilongjiang Medical Science, 37(1):10-12.

[3] Brody H., 2015, Colorectal Cancer. Nature. 521(7551): S1.

[4] Gan L, Yang Y, Li Q, et al., 2018, Epigenetic Regulation of Cancer Progression by EZH2: from Biological Insights to Therapeutic Potential. Biomarker Research, 6(1):10.

[5] Pourjafar M, Samadi P, Karami M, et al., 2020, Assessment of Clinicopathological and Prognostic Relevance of BMI - 1 in Patients with Colorectal Cancer: A Meta - Analysis. Biotechnology and Applied Biochemistry.

[6] Suva ML, Riggi N, Janiszewska M, et al., 2009, EZH2 is Essential for Glioblastoma Cancer Stem Cell Maintenance. Cancer Research, 69(24):9211-9218.

[7] Yu J, Chen L, Bao Z, et al., 2020, BMI1 Promotes Invasion and Metastasis in Endometrial Adenocarcinoma and is a Poor Prognostic Factor. Oncology Reports.

[8] Pan H, Hou J, He J, et al., 2010, Expression and Significance of EZH2 and Bmi-1 Genes in Transitional Cell Carcinoma of the Bladder. Chinese Journal of Experimental Surgery, 27(3):292294.

[9] Shao G, Zhang G, Pu H, et al., 2015, Expression and Clinical Significance of Bmi-1, NANOG, EZH2 in Colorectal Cancer. Journal of Xinjiang Medical University, 38(3): 350-350. 